\title{
Uruguay 1998-2002: la distribución del ingreso en la crisis
}

\author{
Marisa Bucheli y Magdalena Furtado
}

Marisa Bucheli

Investigadora del Departamento de Economía de la Facultad de Ciencias Sociales de la Universidad de la República y consultora de la Oficina de la CEPAL en Montevideo

œmarisa@decon.edu.uy

Magdalena Furtado

Economista del Fondo de Población de las Naciones Unidas (UNFPA) y consultora de la Oficina de la CEPAL en Montevideo

$\infty$ furtado@unfpa.org

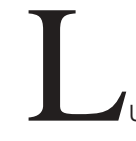

uego de una fase de crecimiento en el decenio de 1990, Uruguay soportó entre 1998 y 2002 una caída del producto de 17,5\%. Este trabajo busca analizar la distribución del ingreso en ese período de crisis y descubrir qué grupos de población fueron los más perjudicados. Los resultados indican que la concentración de ingresos, que había comenzado a mediados del decenio de 1990, se profundizó. Además, el análisis de distintos grupos de población, según características sociodemográficas y socioeconómicas, mostró la continuidad de otros dos fenómenos: el aumento de la diferencia de ingresos entre personas de hogares con distinto nivel educativo, y entre personas de hogares dependientes de jubilaciones y pensiones, por un lado, y de ingresos del trabajo, por otro. Esto se reflejó en la estructura etaria de la población: los menores se vieron más perjudicados que los adultos mayores, consolidándose la tendencia a una creciente concentración de niños en los estratos de ingresos más bajos. 


\section{I}

\section{Introducción}

En la primera mitad del decenio de 1990, cuando en diversos países de la región crecía la desigualdad de los ingresos, Uruguay mantenía una distribución relativamente estable (Vigorito, 1999). Así, era citado como el país latinoamericano con mayor capacidad para amortiguar los efectos sociales adversos de los procesos de apertura, ajustes macroeconómicos y reformas (Kaztman, Filgueira y Furtado, 2000).

Sin embargo, en la segunda mitad de ese decenio comenzó a percibirse un leve aumento de la dispersión de los ingresos. Con la profunda depresión económica que comenzó en 1999, la tendencia concentradora se consolidó. Este trabajo pretende analizar en ese contexto cómo evolucionó la distribución del ingreso entre 1998 y 2002 y, en particular, describir los cambios ocurridos en diferentes grupos de población. Los grupos de población analizados responden tanto a criterios sociodemográficos como socioeconómicos (relativos al origen de los ingresos del hogar), de modo que no solo permiten caracterizar estructuras sociales, sino también explorar las causas de las tendencias de la desigualdad.
En la sección II siguiente se muestra un panorama de mediano plazo de la distribución del ingreso y en la III se aborda un análisis focalizado en el quinquenio 1998-2002. Luego, en la sección IV, se presentan los resultados obtenidos del examen de la desigualdad a partir de la estructura socio-demográfica de la población, y en la $\mathrm{V}$ los obtenidos a partir de su estructura socioeconómica (origen de los ingresos del hogar). Para ello se trabajó con la distribución entre las personas del ingreso per cápita del hogar. Se utilizaron los levantamientos de 1998 y 2002 de la Encuesta Continua de Hogares ( $\mathrm{ECH}$ ) que realiza el Instituto Nacional de Estadística (INE), imputándosele a cada persona el ingreso per cápita de su hogar, incluyendo el valor de la vivienda y excluyendo algunos beneficios sociales. Esto último se debió a que un cambio en el cuestionario de la ECH afectó las medidas de desigualdad, como se detalla en el apéndice A. En el apéndice B se presenta la metodología que se utilizó para analizar los grupos de población.

\section{II}

\section{La década de 1990: crecimiento con indicios de mayor desigualdad}

Desde mediados del decenio de 1980 hasta el año 1998, la actividad económica del país atravesó por una fase de crecimiento, y solo registró una caída $(-1,5 \%)$, que se dio en 1995. Sin embargo, el ingreso per cápita de los hogares presentó una tendencia ascendente solo hasta 1994 y, después de ese año creció únicamente en 1998 (gráfico 1). A menudo se interpreta que la evolución del ingreso per cápita de los hogares respondió menos a la del producto interno bruto (PIB) que a cambios en la tasa de desempleo y al comportamiento del mercado de trabajo en general. En efecto, en 1995 hubo un incremento del desempleo que se acentuó en 1996, a pesar de la recuperación económica; y si bien en el bienio 19971998 la desocupación descendió, se mantuvo en niveles superiores a los de comienzo de la década.
La desigualdad de ingresos se mantuvo estable hasta mediados de la década de 1990 y luego se inició un proceso de concentración de los ingresos que continuó hasta el 2002. Este proceso estuvo en cierta medida relacionado con el hecho de que los ingresos provenientes de jubilaciones y pensiones aumentaron más que aquellos provenientes del trabajo (Bucheli y Rossi, 1994; Machado y Reggio, 1999; Vigorito, 1999; Arim y Furtado, 2000), pero el papel más importante correspondió a los cambios ocurridos en el mercado de trabajo en el decenio de 1990. En efecto, no solamente aumentó el nivel de desempleo en la segunda mitad de esa década, sino que además se acentuó la dispersión de las remuneraciones (Vigorito, 1999; Bucheli y Furtado, 2000a; Kaztman, Filgueira y Furtado, 2000; PNUD, 2001). 
GRÁFICO 1

Uruguay urbano: Variación porcentual anual del ingreso y del PIB per cápita, tasa de desempleo e índice de Gini, 1987-2002
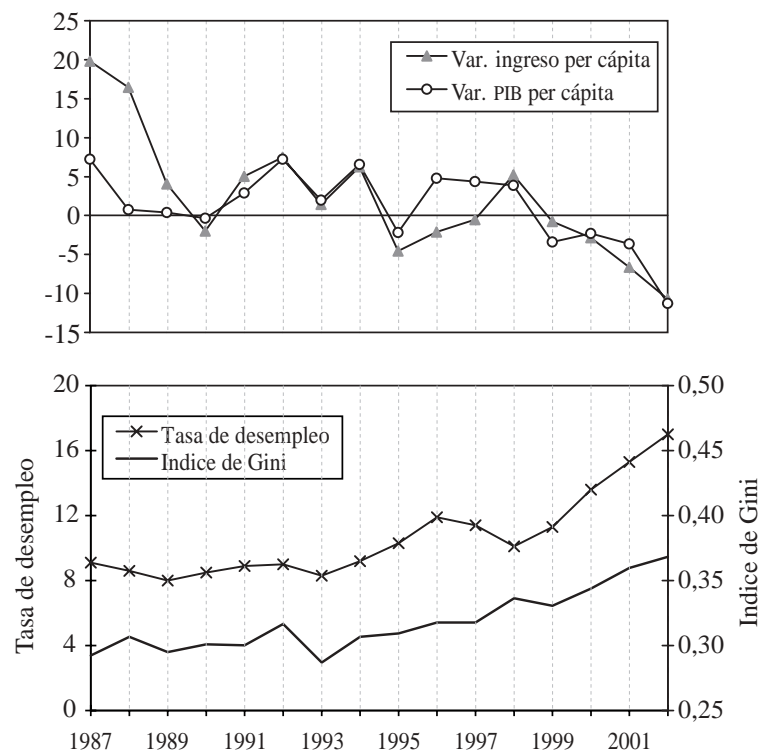

Fuente: Elaboración propia con datos del Banco Central del Uruguay y del Instituto Nacional de Estadística (INE).

Uno de los fenómenos más documentados respecto al aumento de la dispersión de los ingresos del trabajo fue el incremento de la rentabilidad de la educación en la segunda mitad del decenio de 1990. Estimaciones de ecuaciones salariales para los trabajadores del sector privado mostraron hacia fines del decenio un aumento de la rentabilidad derivada de niveles educativos más altos, que amplificaba las diferencias de salarios entre trabajadores con distintos niveles de educación (Bucheli y Furtado, 2000b).

Arim y Zoppolo (2000) encontraron que el aumento de las remuneraciones relativas de los trabajadores con mayores niveles educativos explicó cerca de la mitad del crecimiento de la dispersión, fenómeno consistente con un aumento de la demanda de trabajo calificado más rápido que el crecimiento de su oferta. De acuerdo a Casacuberta y Vaillant (2002), este cambio de la demanda provendría de la introducción de nuevas tecnologías complementarias del trabajo calificado, incentivadas por el proceso de liberalización comercial.

La mayor dispersión de las remuneraciones del trabajo no respondió únicamente al cambio en la rentabilidad de la educación. Arim y Zoppolo (2000) destacan el papel de un segundo factor: la nueva forma de determinar los salarios en la década de 1990, cuan- do se pasó de un régimen de fijación centralizada a nivel de sectores a uno de negociación descentralizada a nivel de empresas.

En efecto, en la última mitad del decenio de 1980 los salarios en cada sector de actividad se determinaban en negociaciones entre el sindicato de trabajadores y la agrupación empresarial correspondiente. La homologación de los convenios por parte del gobierno hacía que el cumplimiento de éstos fuese obligatorio para todas las unidades económicas que realizaban la actividad cubierta por el convenio. Miles y Rossi (2001) señalan que los más favorecidos en esta negociación centralizada eran los trabajadores que se hallaban en la parte baja de la distribución del ingreso, en un período caracterizado por altas tasas de sindicalización. En 1991, el Estado se retiró de esta modalidad de determinación de salarios y la negociación quedó en manos de empresarios y trabajadores, en forma bipartita, pasando a ser libre y voluntaria En el marco de la caída de los niveles de sindicalización y de la implementación de medidas de apertura comercial, este cambio en el papel del Estado habría significado un aumento de la dispersión salarial entre empresas de un mismo sector, con incrementos, por ejemplo, de las diferencias por tamaño, por grado de sindicalización e incluso por niveles educativos.

Un tercer factor que ha contribuido a acentuar la dispersión salarial ha sido la política pública. Miles y Rossi (2001) atribuyen parte del incremento de esa dispersión en el decenio de 1990 al alza de los salarios públicos y la caída del salario mínimo nacional.

Por un lado, el índice de salarios públicos tuvo un crecimiento menor que el de los salarios privados en la primera mitad de la década; pero a partir de 1995 sus variaciones fueron superiores. Así, entre 1995 y 1999 el salario público creció $11 \%$ y el privado $2 \%$. Utilizando las encuestas de hogares de principios y fines de la década de 1990, Miles y Rossi encontraron que en cada cuantil de la distribución de los salarios públicos y privados, los primeros subieron relativamente más o cayeron menos que los segundos, lo que se tradujo en un aumento de la dispersión global de salarios.

Por otro lado, en esa década el salario mínimo presentó una tendencia decreciente en términos reales. Esta evolución no repercutió en la capital del país, donde vive poco menos de la mitad de la población, ya que el proceso de negociación permitió a los trabajadores obtener salarios mínimos por encima del oficial. En cambio, afectó al resto del país urbano - donde las remuneraciones han sido tradicionalmente menores que 
en la capital- pues la dispersión geográfica de los trabajadores facilitó a las empresas el ejercicio de un poder monopsónico. Como consecuencia, la caída del salario mínimo habría impulsado una fuerte disminución de las remuneraciones reales en los estratos más bajos de esas áreas.

\section{III}

\section{Los años 1998-2002: crisis con profundización de la desigualdad}

La evolución de los indicadores presentada en el gráfico 1 refleja la crisis reciente: la caída de la actividad económica se tradujo en una disminución de los ingresos de los hogares y en un aumento de la tasa de desempleo.

En efecto, en 1999 comenzó una recesión económica que se profundizó en los años siguientes, acumulándose entre 1998 y 2002 una caída del producto del orden del $17,5 \%$. La baja de la actividad comenzó asociada a la pérdida de competitividad con respecto a Brasil ocasionada por la devaluación de la moneda en ese país, pérdida que se sumó a la que ya venía experimentando Uruguay con respecto al resto del mundo. Los efectos se agravaron debido a la conjunción de otros factores: el alza de la tasa de interés internacional y del precio del petróleo, la caída de los precios internacionales de ciertos bienes exportados por Uruguay (carne, lana y arroz, entre otros), una sequía con impactos especialmente severos en los sectores agropecuario y de generación de electricidad, y la aparición de la fiebre aftosa a principios del 2001, lo que implicó el cierre de varios mercados de destino de las exportaciones uruguayas. A todo esto se sumaron, por último, los efectos del abrupto abandono de la convertibilidad y el estallido de la crisis financiera en Argentina, seguidos por la crisis financiera en Uruguay, que culminó en 2002 con una caída del producto de $10,8 \%$.

En estas circunstancias, la tasa de desempleo trepó hasta alcanzar sus máximos niveles históricos, arrojando un promedio de $17 \%$ en 2002 , al tiempo que creció el desempleo de larga duración. A la vez, descendieron en términos reales los valores medios de las jubilaciones y pensiones, los ingresos del trabajo y los del capital.

La merma de los ingresos provenientes del capital puede asociarse a la crisis financiera, que, entre otras cosas, provocó el descenso de los intereses, el cierre de instituciones financieras y la baja de los precios de los alquileres debido al menor volumen de negocios inmobiliarios.

La caída de las jubilaciones y pensiones se relacionó en gran medida con una modificación efectuada el 2002 al Impuesto a las Retribuciones Personales (IRP). Este impuesto, que grava tanto a las jubilaciones y pensiones como a los salarios, se caracteriza por tener tasas crecientes para franjas de retribuciones. Con la modificación, el número de franjas se incrementó y el rango de tasas se amplió, causando una baja de las jubilaciones y pensiones netas.

Obviamente, esta modificación también influyó en el ingreso disponible de los trabajadores del sector formal. Sin embargo, el descenso de los ingresos del trabajo estuvo más bien relacionado con la crisis y la dinámica del mercado laboral, asistiéndose además a un aumento de la dispersión de los salarios. Esta continuidad del crecimiento de la concentración de los ingresos laborales tuvo características semejantes a las señaladas para la segunda mitad del decenio de 1990.

Por un lado, el desfase entre el índice de los salarios públicos y el de los privados continuó. Si hasta 1999 los primeros habían subido más que los segundos, a partir de entonces cayeron menos. Así, entre 1998 y 2002 los salarios públicos acumularon una caída de $8 \%$ en términos reales y los salarios privados una de $12 \%$, lo que indica que si bien los trabajadores públicos no fueron inmunes a la crisis, tuvieron cierta "protección".

Por otro lado, continuó el aumento de las diferencias salariales vinculadas a disparidades de niveles educativos (Amarante y Arim, 2003). Además, si bien la desocupación afectó a todos los trabajadores, la probabilidad de estar desempleado creció menos para los de nivel educativo más alto (Bucheli y Casacuberta, 2003). Desde el punto de vista del empleo con cober- 
tura de la seguridad social, no es posible analizar las tendencias debido a una modificación en el relevamiento de la información en el año 2001. Sin embargo, es probable que la tendencia a un mayor crecimiento de la actividad informal entre los trabajadores con menor nivel educativo registrada en el decenio de 1990 haya continuado en los años 2001 y 2002: ante el impacto diferenciado del desempleo, puede haber respondido a una estrategia de sobrevivencia de los trabajadores ante la crisis. De hecho, entre 1998 y 2002 aumentó la participación de los trabajadores por cuenta propia sin local, los que elevaron su participación en el empleo del $7 \%$ al $10 \%$.

En este contexto general, entre 1998 y 2002 el ingreso per cápita medio cayó $20 \%$ y el proceso de concentración de los ingresos continuó. El índice de Gini —calculado para la distribución entre las personas del ingreso per cápita del hogar — subió de 0,437 a 0,459, el índice de Theil de 0,344 a 0,382 y el índice de entropía 0 de 0,336 a 0,367 .

\section{IV}

\section{Los cambios en la estructura sociodemográfica}

Las posiciones de diversos grupos sociodemográficos han sido extensamente estudiadas en el país. Los niños y adolescentes, los hogares extendidos y los hogares formados por adultos de bajo nivel educativo han estado tradicionalmente sobrerrepresentados en los estratos de menores ingresos. Esto se refleja en el cuadro 1, donde se presenta la participación de los distintos grupos en los quintiles de la distribución del ingreso per cápita de las personas.

Así, el quintil 1 se configuró con $40 \%$ de menores de 14 años y sólo 4\% de mayores de 59; el quintil 5 en cambio, se compuso de $35 \%$ de adultos mayores y sólo $9 \%$ de niños. Mientras que el $31 \%$ de la población vivía en hogares cuyo jefe era mayor de 59 años, este grupo representó el $13 \%$ del quintil 1 y el $43 \%$ del quintil 5. En cuanto al tipo de hogar, la proporción de personas que vivían solas o únicamente con su pareja — situación asociada a las edades mayores- fue superior en los estratos altos que en los bajos: en efecto, aunque constituían $17 \%$ de la población total, fueron el $37 \%$ del quintil 5.

Por último, existe una clara relación entre el nivel educativo del jefe del hogar y la ubicación de las personas en la escala de ingresos. El $43 \%$ de las personas vivía en hogares cuyo jefe tenía educación primaria: esta proporción alcanzó el $61 \%$ en el quintil 1 y fue solo de $21 \%$ en el quintil 5 . En el otro extremo, no se registraron miembros de hogares con jefe de educación terciaria completa en el quintil 1, en tanto que este grupo representó la cuarta parte de la población en el quintil 5.
El cuadro 2 exhibe la descomposición de los índices de entropía 0 y 1 para los años 1998 y 2002, la que permite conocer el poder explicativo de las características presentadas. El componente "dentro" recoge la contribución de la desigualdad interna de los grupos; el componente "entre" refleja la contribución de la desigualdad entre grupos (apéndice B). Los resultados obtenidos indican que el nivel educativo del jefe de hogar fue la característica que dio lugar a la clasificación con mayor desigualdad entre los grupos. Más aún, su poder explicativo creció entre 1998 y 2002, lo que condice con la información que se presenta en el cuadro 3 .

La participación en la población de los distintos grupos sociodemográficos ha experimentado algunos cambios en el largo plazo, como una mayor incidencia de niveles educativos más altos, de jefaturas de hogar femeninas y de hogares monoparentales. Pero la magnitud de esos cambios resulta pequeña al comparar 1998 y 2002 (cuadro 3). Al mismo tiempo, las variaciones de los índices de desigualdad de cada grupo generalmente indican aumento - esto es, mayor concentración-, lo que significa que cada una de las cuatro clasificaciones analizadas en el cuadro tendió a tornarse menos homogénea.

Con respecto a la variación del ingreso per cápita medio, se observa un aumento de la distancia entre los grupos. En efecto, si bien para todas las clasificaciones disminuyeron los ingresos medios de todos los grupos, en algunos grupos cayeron más (cuadro 3): particularmente, en los menores de 14 años, las parejas con hijos 
CUADRO 1

Uruguay urbano: Participación de los distintos grupos sociodemográficos en los quintiles y en el total de la población, $2002^{a}$

(Porcentajes)

\begin{tabular}{llllllll}
\hline & Quintil 1 & Quintil 2 & Quintil 3 & Quintil 4 & Quintil 5 & Total \\
\hline
\end{tabular}

Edad de las personas

0 a 13 años
14 a 20
21 a 59
60 o más

Sexo y edad del jefe de hogar

Hombre $<60$

Hombre $>=60$

Mujer $<60$

Mujer $>=60$

Tipo de hogar

Unipersonal

Pareja sin hijos

Pareja con hijos

Monoparental

Otro

Educación del jefe de hogar

Primaria

Media incompleta

Media completa

Terciaria incompleta

Terciaria completa

Sin datos

\begin{tabular}{rr|rrrr}
40 & 26 & 18 & 12 & 9 & 21 \\
15 & 14 & 11 & 8 & 7 & 11 \\
40 & 49 & 50 & 49 & 50 & 48 \\
4 & 12 & 21 & 31 & 35 & 21 \\
100 & 100 & 100 & 100 & 100 & 100
\end{tabular}

\begin{tabular}{|rrrrrr|}
\hline 67 & 61 & 53 & 46 & 43 & 54 \\
\hline 9 & 15 & 23 & 26 & 26 & 20 \\
20 & 16 & 13 & 14 & 14 & 15 \\
\hline 4 & 8 & 11 & 14 & 17 & 11 \\
100 & 100 & 100 & 100 & 100 & 100 \\
\hline
\end{tabular}

\begin{tabular}{|rrrrrr}
0 & 1 & 3 & 8 & 17 & 6 \\
1 & 4 & 10 & 18 & 20 & 11 \\
56 & 51 & 45 & 38 & 38 & 45 \\
8 & 8 & 10 & 9 & 10 & 9 \\
35 & 36 & 33 & 27 & 16 & 29 \\
100 & 100 & 100 & 100 & 100 & 100
\end{tabular}

\begin{tabular}{rrrrrr}
61 & 49 & 47 & 38 & 21 & 43 \\
29 & 35 & 31 & 29 & 23 & 29 \\
7 & 11 & 14 & 17 & 19 & 14 \\
0 & 2 & 3 & 6 & 11 & 5 \\
0 & 1 & 3 & 8 & 25 & 8 \\
2 & 2 & 2 & 1 & 0 & 2 \\
100 & 100 & 100 & 100 & 100 & 100 \\
\hline
\end{tabular}

Fuente: Elaboración propia con datos de la Encuesta Continua de Hogares del Instituto Nacional de Estadísticas (INE).

a Las cifras resaltadas en gris corresponden a las celdas en que el respectivo grupo tiene mayor representación en el quintil que en el total de la población.

y los hogares con jefe de nivel educativo más bajo. Obsérvese que estos grupos son los que estructuralmente estaban desde el inicio en las peores ubicaciones de la distribución. Así, si se analiza por tramo de edad, la caída del ingreso fue de $15 \%$ para los mayores de 59 años, mientras que fue del $21 \%$ y $24 \%$ para los menores de 14 años y los de 14 a 20 años, respectivamente. A su vez, si analiza por nivel educativo del jefe de hogar, la mayor caída afectó al grupo con jefe con educación media incompleta (-27\%), seguido del gru- po con jefe con educación primaria (-21\%). En el otro extremo, los menos desfavorecidos en el período fueron los integrantes de hogares con jefe con educación terciaria completa, cuyos ingresos cayeron sólo un $12 \%$, seguidos por aquellos con jefe con educación terciaria incompleta (-18\%). Por lo tanto, la evolución en el quinquenio amplió aún más las brechas medias de ingreso por nivel educativo del jefe de hogar, lo que se reflejó en el mencionado aumento del poder explicativo de esta clasificación. 
CUADRO 2

Uruguay urbano: Descomposición de los índices de entropía 0 y 1 en dos componentes: la contribución a la desigualdad total de la desigualdad dentro de los grupos sociodemográficos y entre dichos grupos, 1998 y 2002 (Porcentajes)

\begin{tabular}{llll}
\multicolumn{2}{c}{ Entropía 0 } & 2002 & \multicolumn{3}{c}{ Entropía 1 } \\
\cline { 3 - 4 } & 1998 & 2002
\end{tabular}

Edad de las personas

Desigualdad dentro de los grupos

Desigualdad entre los grupos

92

8

98

Desigualdad entre los grupos

Tipo de hogar

Desigualdad dentro de los grupos

Desigualdad entre los grupos

Educación del jefe de hogar

Desigualdad dentro de los grupos

Desigualdad entre los grupos

Fuente: Elaboración propia con datos de la Encuesta Continua de Hogares del Instituto Nacional de Estadísticas (INE).

Uruguay urbano: Variación de la participación en la población, del ingreso per cápita medio y de la desigualdad de los distintos grupos sociodemográficos, entre 1998 y 2002

\begin{tabular}{|c|c|c|c|c|}
\hline & $\begin{array}{l}\text { Variación de la } \\
\text { participación en } \\
\text { la población (\%) }\end{array}$ & $\begin{array}{l}\text { Variación del } \\
\text { ingreso per cápita } \\
\text { medio }(\%)\end{array}$ & $\begin{array}{c}\text { Variación del } \\
\text { índice de Entropía } 0 \\
\text { (puntos porcentuales) }\end{array}$ & $\begin{array}{c}\text { Variación del } \\
\text { índice de Entropía } 1 \\
\text { (puntos porcentuales) }\end{array}$ \\
\hline Toda la población & & -20 & 3,1 & 3,8 \\
\hline \multicolumn{5}{|l|}{ Edad de las personas } \\
\hline 0 a 13 & -3 & -24 & 1,8 & 5,1 \\
\hline 14 a 20 & -3 & -21 & 3,6 & 4,8 \\
\hline 21 a 59 & 0 & -23 & 3,2 & 4,0 \\
\hline 60 o más & 9 & -15 & 0,4 & 0,8 \\
\hline \multicolumn{5}{|c|}{ Sexo y edad del jefe de hogar } \\
\hline Hombre<60 & -7 & -22 & 3,9 & 5,6 \\
\hline Hombre $>=60$ & 6 & -18 & $-0,2$ & 0,5 \\
\hline Mujer $<60$ & 16 & -24 & 1,7 & 3,5 \\
\hline Mujer $>=60$ & 12 & -14 & 2,6 & 1,5 \\
\hline \multicolumn{5}{|l|}{ Tipo de hogar } \\
\hline Unipersonal & 19 & -12 & 0,1 & 1,0 \\
\hline Pareja sin hijos & 8 & -21 & $-1,3$ & $-0,4$ \\
\hline Pareja con hijos & -6 & -22 & 3,9 & 5,2 \\
\hline Monoparental & 5 & -22 & $-0,9$ & $-0,9$ \\
\hline Otro & 4 & -22 & 1,9 & 3,0 \\
\hline \multicolumn{5}{|c|}{ Educación del jefe de hogar } \\
\hline Primaria & -6 & -21 & 1,3 & 1,4 \\
\hline Media incompleta & 8 & -27 & 2,3 & 3,3 \\
\hline Media completa & 7 & -19 & 1,5 & 1,0 \\
\hline Terciaria incompleta & -5 & -18 & 2,8 & 2,4 \\
\hline Terciaria completa & 3 & -12 & 2,3 & 2,3 \\
\hline
\end{tabular}

Fuente: Elaboración propia con datos de la Encuesta Continua de Hogares del Instituto Nacional de Estadísticas (INE). 


\section{V}

\section{Los cambios en la estructura socioeconómica: la distribución y el origen de los ingresos}

En esta sección se analiza la desigualdad basada en el origen de los ingresos del hogar. Para ello, se clasifica a la población según los siguientes criterios: por un lado, la fuente principal del ingreso con que el hogar se sustenta y el nivel educativo del jefe de hogar, y por otro, el tipo de actividad de los perceptores de ingreso.

En el primer caso se divide a las personas en diez grupos: uno es el de personas en hogares donde más del $65 \%$ del ingreso proviene de una fuente claramente identificada — remuneraciones del trabajo, ingresos del capital, o jubilaciones y pensiones-, y los restantes corresponden a personas cuyos ingresos dependen de combinaciones de distintas fuentes. Para quienes viven del trabajo y de jubilaciones y pensiones, se toma en cuenta además el nivel educativo del jefe de hogar, distinguiendo entre educación primaria, media y terciaria.

En el segundo caso, se distingue entre las personas que viven en hogares compuestos únicamente por trabajadores, por propietarios del capital (ya sea patrones o rentistas), o por pasivos (ya sea jubilados o pensionados). También se consideran combinaciones de trabajadores y pasivos y, finalmente, combinaciones de propietarios del capital con trabajadores o con jubilados. Adicionalmente, se considera la presencia de desocupados (exceptuados quienes buscan trabajo por primera vez) dentro de dos grupos de hogares: en los compuestos únicamente por trabajadores y en los que combinan trabajadores con pasivos.

Como se observa en el cuadro 4, la primera de estas clasificaciones fue la que contribuyó en mayor medida a explicar la desigualdad de ingresos.

\section{Fuente principal del ingreso del hogar y nivel educativo del jefe de hogar}

En todo el quinquenio, la principal fuente de ingresos fue la remuneración del trabajo. Por ejemplo, en el año 2002 el 54\% de las personas vivía en hogares donde más del 65\% de los ingresos se originaba en el mercado de trabajo. En cambio, el 13\% de las personas dependía de jubilaciones o pensiones y tan sólo el 3\%, de ingresos provenientes del capital. El resto de las personas dependía de la combinación de distintas fuentes de ingreso (cuadro 5)

La ubicación de los grupos refleja la relación entre los ingresos y el nivel educativo del jefe de hogar. Además, se aprecia que quienes dependían de jubilaciones y pensiones estaban en mejor posición que quienes se sustentaban de ingresos del trabajo. Así, las personas en hogares que tenían jefe con educación primaria y que dependían del trabajo estuvieron sobrerrepresentadas en los quintiles 1 y 2 , mientras que las dependientes de jubilaciones y pensiones se ubicaron en mayor medida en los quintiles 3 y 4 . En el caso de hogares con jefe de nivel educativo medio, si la fuente principal era el trabajo, el grupo quedó sobrerrepresentado en los quintiles 2 a 4; en cambio, si la fuente principal era la jubilación, la sobrerrepresentación se dio en los quintiles 4 y 5 . Finalmente, si el jefe tenía nivel educativo terciario, hubo sobrerrepresentación en los quintiles 4 y 5 cuando los ingresos dependían del trabajo y solamente en el quintil 5 cuando dependían de jubilaciones y pensiones.

Por otra parte, las personas con ingresos dependientes del capital estuvieron sobrerrepresentadas en los quintiles 4 y 5 , conformando entonces el estrato alto junto con los grupos cuyos jefes tenían nivel educativo superior.

El cuadro 6 muestra las variaciones de la participación de grupos socioeconómicos en la población, y la variación de sus ingresos per cápita medios y de sus índices de distribución. A diferencia de las clasificaciones sociodemográficas de la sección anterior, ésta recoge importantes cambios en la estructura por grupos de población. En particular, aumentó en un $70 \%$ la proporción de personas en hogares con múltiples fuentes de ingreso ("resto"), pasando del $8 \%$ al $14 \%$ de la población entre 1998 y 2002. Esto ocurrió particularmente en los quintiles 1 y 2 , lo cual podría estar reflejando la fusión de hogares como forma de enfrentar los efectos de la crisis. Además, creció la proporción de hogares que cuenta con ayuda familiar y/o subsidios públicos, en particular seguro de desempleo. 
CUADRO 4

Uruguay urbano: Descomposición de los índices de entropía 0 y 1

en dos componentes: la contribución a la desigualdad total de la

desigualdad dentro de los grupos socioeconómicos, y de la desigualdad

entre dichos grupos, 1998 y 2002

(Porcentajes)

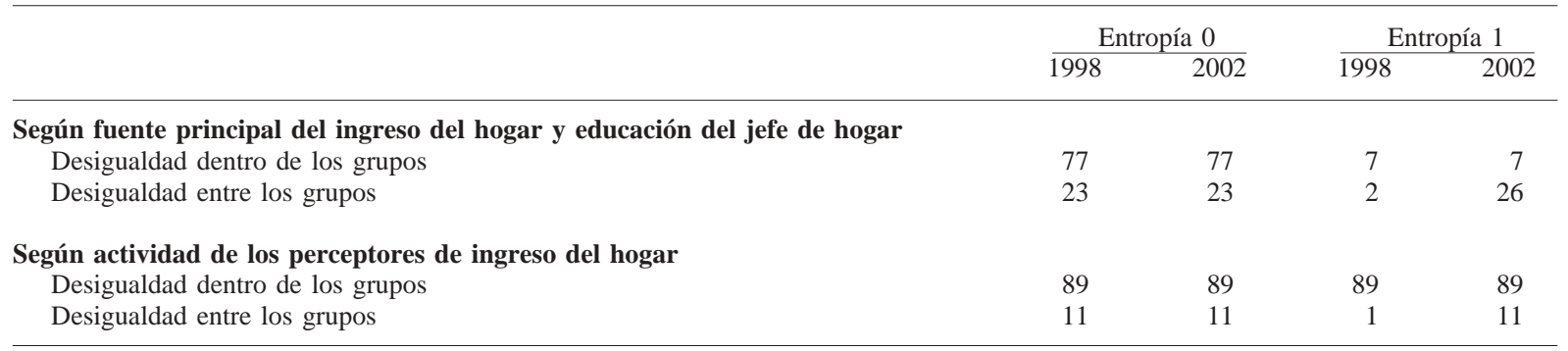

Fuente: Elaboración propia con datos de la Encuesta Continua de Hogares del Instituto Nacional de Estadística (INE).

CUADRO 5

Uruguay urbano: Participación de los grupos socioeconómicos en los quintiles y en el total de la población, 2002a

(Porcentajes)

\section{Quintil 1 Quintil 2 Quintil 3 Quintil 4 Quintil 5 Total}

Fuente principal del ingreso del hogar y nivel

educativo del jefe de hogar

Trabajo de jefe con educación primaria

Trabajo de jefe con educación media

Trabajo de jefe con educación terciaria

Jubilación de jefe con educación primaria

Jubilación de jefe con educación media

Jubilación de jefe con educación terciaria

Capital

Trabajo y jubilación

Capital y jubilación

Resto

$\begin{array}{rrrrrr}33 & 26 & 21 & 13 & 6 & 20 \\ 24 & 33 & 31 & 29 & 20 & 27 \\ 0 & 2 & 4 & 9 & 21 & 7 \\ 4 & 7 & 11 & 14 & 9 & 9 \\ 1 & 2 & 3 & 5 & 8 & 3 \\ 0 & 0 & 0 & 1 & 5 & 1 \\ 1 & 1 & 2 & 5 & 8 & 3 \\ 9 & 15 & 17 & 15 & 10 & 13 \\ 0 & 0 & 0 & 1 & 3 & 1 \\ 29 & 14 & 10 & 8 & 10 & 14 \\ 100 & 100 & 100 & 100 & 100 & 100\end{array}$

Actividad de los perceptores de ingreso del hogar

Trabajadores, sin desocupados

Trabajadores, con desocupados

Patrones o rentistas

Jubilados o pensionados

Trabajadores y jubilados, sin desocupados

Trabajadores y jubilados, con desocupados

Patrones o rentistas (y jub. o trab.)

Resto

\begin{tabular}{|rr|}
49 & 45 \\
27 & 20 \\
\hline 0 & 0 \\
4 & 8 \\
8 & 16 \\
\hline 5 & 7 \\
\hline 1 & 1 \\
6 & 3 \\
100 & 100 \\
\hline
\end{tabular}

$\begin{array}{rrrr}44 & 40 & 41 & 44 \\ 13 & 8 & 5 & 15 \\ 1 & 2 & 3 & 1 \\ 13 & 19 & 22 & 13 \\ 20 & 22 & 16 & 16 \\ 6 & 3 & 1 & 4 \\ 3 & 5 & 10 & 4 \\ 2 & 2 & 2 & 3 \\ 100 & 100 & 100 & 100\end{array}$

Fuente: Elaboración propia con datos de la Encuesta Continua de Hogares del Instituto Nacional de Estadísticas (INE).

a Las cifras resaltadas en gris corresponden a las celdas donde el grupo respectivo tiene mayor representación en el quintil que en el total de la población. 


\begin{tabular}{lccc}
\hline & $\begin{array}{c}\text { Variación de la } \\
\text { participación en } \\
\text { la población }(\%)\end{array}$ & $\begin{array}{c}\text { Variación del } \\
\text { ingreso per cápita } \\
\text { medio }(\%)\end{array}$ & $\begin{array}{c}\text { Variación del } \\
\text { índice de Entropía 0 } \\
\text { (puntos porcentuales) }\end{array}$ \\
\hline Toda la población & $-20 \%$ & $\begin{array}{c}\text { Variación del de Entropía 1 } \\
\text { (puntos porcentuales) }\end{array}$
\end{tabular}

Fuente principal del ingreso del hogar y nivel educativo del jefe de hogar Trabajo jefe con educación primaria

Trabajo jefe con educación media

Trabajo jefe con educación terciaria

Jubilación jefe con educación primaria

Jubilación jefe con educación media

Jubilación jefe con educación terciaria

Capital

Trabajo y jubilación

Capital y jubilación

Resto

$\begin{array}{rr}-24 & -23 \\ -3 & -24 \\ 1 & -10 \\ 12 & -10 \\ 39 & -12 \\ 10 & -11 \\ -24 & -24 \\ 1 & -17 \\ -9 & -12 \\ 70 & -41\end{array}$

$-0,2$
1,7
4,6
0,2
2,0
$-1,3$
0,4
$-1,8$
0,1
3,4

1,0
1,9
5,0
0,6
3,4
$-1,7$
4,4
$-0,8$
$-0,7$
8,0

Actividad de los perceptores de ingreso del hogar

Trabajadores, sin desocupados

Trabajadores, con desocupados

Patrones o rentistas

Jubilados o pensionados

Trabajadores y jubilados sin desocupados

Trabajadores y jubilados con desocupados

Patr. O rent. y jub. o trab.

Resto

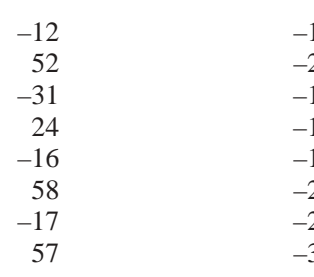

$\begin{array}{rrr}-17 & 4,8 & 6,4 \\ -22 & 3,0 & 5,6 \\ -18 & 3,8 & 7,2 \\ -12 & 1,1 & 1,9 \\ -13 & -0,2 & 0,7 \\ -22 & -2,9 & -2,7 \\ -23 & -0,9 & -0,5 \\ -38 & 2,7 & 4,9\end{array}$

Fuente: Elaboración propia con datos de la Encuesta Continua de Hogares del Instituto Nacional de Estadística (INE).

Con respecto a las variaciones del ingreso per cápita medio de los grupos, cabe destacar ante todo que el aumento de las diferencias de ingreso por nivel educativo señaladas en la sección anterior se dio solamente en el grupo de dependientes del trabajo. En cambio, para quienes vivían de jubilaciones y pensiones y estaban más sujetos a decisiones institucionales que a las fluctuaciones económicas, la variación de los ingresos fue independiente del nivel educativo del jefe de hogar, registrándose caídas de entre $10 \%$ y 12\% para los tres niveles educativos.

Para las personas en hogares dependientes del trabajo, el ingreso per cápita medio cayó $10 \%$ cuando el jefe de hogar tenía educación terciaria y entre $23 \%$ y $24 \%$ cuando tenía nivel educativo bajo o medio. Recuérdese que en el mercado de trabajo hubo algunos cambios que pueden contribuir a explicar este distanciamiento: por un lado, el desempleo afectó en mayor medida a los trabajadores de menor nivel educativo y por el otro, la actividad informal creció en esos sectores, probablemente como una estrategia de refugio. Ambos factores tendieron a disminuir el ingreso de esta población. Obsérvese, por otra parte, que dentro de cada grupo de dependientes del trabajo la desigualdad creció: esto sugiere que otros factores, además de la diferenciación entre niveles educativos, tendieron a concentrar las remuneraciones laborales.

Los movimientos en los ingresos per cápita medios produjeron un segundo fenómeno de importancia: para el nivel educativo bajo y medio, la disparidad entre los dependientes del trabajo y los dependientes de jubilaciones y pensiones tendió a crecer. Así, dadas las posiciones iniciales de los grupos, la fuente de ingresos tendió a distanciarlos.

El tercer fenómeno de interés fue la importante pérdida en términos relativos de los miembros de hogares dependientes de ingresos del capital: sus ingresos disminuyeron alrededor del 24\% entre 1998 y 2002, lo que puede asociarse a la crisis financiera. Así, las mayores pérdidas afectaron a grupos que estaban en los dos extremos de la distribución. 


\section{Actividad de los perceptores de ingresos del hogar}

La ubicación relativa de los grupos según la actividad de los perceptores de ingreso es consistente con los resultados de la clasificación anterior. Así, por un lado, las personas en hogares de trabajadores estuvieron sobrerrepresentadas en los estratos más pobres; por otro lado, las que vivían en hogares con perceptores de ingresos del capital y/o de jubilaciones y pensiones tendieron a ubicarse en los estratos más altos.

La novedad de la clasificación según este criterio reside en que considera la presencia de desocupados en el hogar: los miembros de hogares con desocupados se situaron en una posición más desfavorable. Puesto que el concepto de desocupado que se utilizó no incluye a quienes buscan trabajo por primera vez, este resultado no sorprende: básicamente, se trata de hogares que perdieron un perceptor de ingresos durante la crisis. El ingreso per cápita medio de los integrantes de estos hogares no solamente fue el menor en esta clasificación sino que también en relación con cualquier otro grupo del resto de las clasificaciones realizadas. Por otra parte, los índices de desigualdad interna de tales hogares fueron relativamente bajos en comparación al resto. Así, se trata de hogares en situaciones deprimidas que además exhiben una relativa homogeneidad interna.

La distancia entre los ingresos de las personas en hogares con al menos un desocupado y los de otros grupos tendió a ampliarse. Así, en los hogares de trabajadores el ingreso per cápita medio disminuyó $17 \%$ cuando no había desocupados y $22 \%$ cuando había al menos uno. Para las personas dependientes de jubilaciones y pensiones o en hogares que combinaban pasivos y trabajadores sin desocupados, el ingreso cayó entre $12 \%$ y $13 \%$. Por último, los hogares de pasivos con presencia de desocupados arrojaron una caída del ingreso de $22 \%$.

El aumento de la distancia entre los ingresos de los miembros de hogares con desocupados y los de otros grupos podría obedecer a que en el 2002 esos desocupados tenían una posición relevante como perceptores de ingresos en el hogar. Algunos indicadores sugieren que esta explicación podría ser importante: en 1998, en el $17 \%$ de los hogares de "trabajadores con desocupados" el que estaba desempleado era el jefe de hogar; en el año 2002 esto sucedió en el $22 \%$ de los casos. Así, en el 2002 los hogares con desocupados se habrían visto afectados en mayor medida por la pérdida del ingreso de su principal sostén; además, es posi- ble que el nivel educativo de los hogares con desocupados haya sido más bajo en el 2002 que en 1998. Puesto que el desempleo tendió a afectar más a las personas de bajo nivel educativo, y dado que el nivel educativo era homogéneo dentro de los hogares y que los ingresos tenían una correlación positiva con los años de educación alcanzados, el mayor aumento del desempleo de los menos calificados podría reflejarse en un menor ingreso per cápita de los hogares con desocupados en el año 2002.

Finalmente, con la crisis creció el porcentaje de personas en hogares de trabajadores con al menos un desocupado (del 10\% en 1998 al $15 \%$ en 2002). Los resultados, pese al bajo número de casos, también sugieren un leve incremento tanto de la proporción de personas en hogares con desocupados que combinan trabajadores y jubilados o pensionados, como del "resto", donde es alto el peso de los subsidios. Esto es coherente con una caída del empleo privado en el período de alrededor de 80.000 puestos de trabajo y con la política de no contratación de funcionarios públicos (Amarante y Arim, 2003).

\section{Síntesis de los cambios}

Para analizar el impacto de los cambios relacionados con el origen de los ingresos, se descompuso la variación de la desigualdad total medida a través del índice de entropía 0 en los componentes que contribuyen a explicarla. Estos componentes refieren a los cambios en la desigualdad interna de los grupos, las modificaciones en la estructura de la población entre grupos y las variaciones de las diferencias de ingreso per cápita medio de los grupos (apéndice 2). Los resultados obtenidos aparecen en el cuadro 7.

La variación del índice de entropía 0 entre 1998 y 2002 fue de 3,1 puntos porcentuales. El impacto neto del aumento de la desigualdad dentro de los grupos es recogido en el término $\mathrm{A}$, donde el signo positivo indica un impacto concentrador. En la clasificación por tipo de perceptor, este componente fue primordial para explicar el incremento de la concentración. Por lo tanto, dicha clasificación en sí dio cuenta de una parte menor de los cambios y, en sentido estricto, perdió poder explicativo, ya que obviamente algunas variables no consideradas en ella habrían adquirido importancia durante el período.

En el apartado anterior se mencionó, sin embargo, el importante traspaso de población hacia el grupo de personas en hogares con al menos un desocupado, lo que debería haber contribuido a acrecentar la proporción de personas en el extremo inferior de la 


\begin{tabular}{lcc}
\hline & $\begin{array}{c}\text { Fuente principal de ingreso y educación } \\
\text { del jefe de hogar }\end{array}$ & $\begin{array}{c}\text { Actividad de los perceptores } \\
\text { de ingreso del hogar }\end{array}$ \\
\hline Variación del índice de entropía 0 & 3,1 & 3,1 \\
& & 2,7 \\
Componente de desigualdad dentro de los grupos & 1,0 & $-0,3$ \\
Término A & 1,0 & 2,4 \\
Término B & 2,0 & 0,3 \\
Subtotal & & 0,3 \\
Componente de desigualdad entre los grupos & $-0,8$ & 0,7 \\
Término C & 2,0 & 1,2 \\
Término D & 1,2 & \\
Subtotal & & \\
\hline
\end{tabular}

Fuente: Elaboración propia con datos de la Encuesta Continua de Hogares del Instituto Nacional de Estadística (INE).

distribución. Los efectos de los traspasos se recogen en los términos $\mathrm{B}$ y $\mathrm{C}$ del cuadro 7. El signo negativo del término B se debió a que los cambios en la composición de la población se dieron desde grupos con alta concentración hacia grupos con mayor igualdad interna. Recuérdese que los grupos que incluían desempleados fueron los que registraron los menores índices de desigualdad, indicando situaciones deprimidas y de relativa homogeneidad interna. Por tal vía, entonces, estos traslados tuvieron un efecto neto desconcentrador. En cambio, la vía a través del término $\mathrm{C}$ tuvo un impacto concentrador: esta vía es la que refleja traspasos de personas desde los estratos medios hacia los altos o bajos. Obsérvese que si bien el signo del término fue positivo, el efecto neto de los traspasos fue nulo.

\section{VI}

\section{Conclusiones}

Uruguay transitó por un período de crecimiento con indicios de mayor desigualdad hacia fines de la década de 1990. La creciente desigualdad estuvo en gran medida relacionada con cambios ocurridos en el mercado de trabajo, que registró un mayor nivel de desempleo en la segunda mitad de la década, y con un aumento de la dispersión de las remuneraciones.

Tres fueron los fenómenos más documentados con respecto al aumento de la dispersión de los ingresos
Por último, el término D recoge el impacto de los cambios en las diferencias de ingresos per cápita medios de los grupos. Su signo positivo en ambas columnas del cuadro 7 indica un impacto concentrador y su considerable valor en la segunda columna refleja el importante efecto del incremento de las brechas de ingreso sobre el aumento de la desigualdad total. En particular, el término D refleja lo ocurrido con la relación entre los ingresos por jubilaciones y pensiones y los ingresos del trabajo y, dentro de los ingresos del trabajo, con los vinculados a la educación terciaria versus la educación media o baja. No obstante, en dicho cuadro también se observa un efecto concentrador del aumento de la desigualdad dentro de los grupos. del trabajo en el decenio de 1990: i) el aumento de las diferencias de salarios entre trabajadores con distinto nivel educativo; ii) el cambio en la negociación salarial, la que pasó de un régimen de fijación centralizada a nivel de sectores a uno descentralizado a nivel de la empresa, y iii) la participación del gobierno en el mercado de trabajo, en particular por el mayor aumento de los salarios públicos en relación con los privados, y la caída del salario mínimo nacional. 
En 1999 comenzó un período de recesión económica que se profundizó en los años siguientes, acumulándose entre 1998 y 2002 una caída del producto del orden del $17,5 \%$. La tasa de desempleo subió hasta alcanzar sus máximos niveles históricos, arrojando un promedio de $17 \%$ en el 2002; el desempleo de larga duración aumentó, y los valores medios de los ingresos del trabajo y del capital y de las jubilaciones y pensiones disminuyeron en términos reales. Además de descender las remuneraciones, continuó incrementándose la dispersión salarial, en un marco de aumento de las brechas salariales entre niveles educativos y un mayor crecimiento de los salarios públicos con respecto a los privados.

En este período de crisis bajaron los ingresos de la población en general y se profundizó la desigualdad.

El análisis de diversas clasificaciones sociodemográficas — por edad, por sexo y edad del jefe de hogar, por tipo de hogar y por educación del jefe- mostró un empeoramiento de la situación de todos los grupos. No obstante, fueron algunos los que se vieron más perjudicados: los menores, las parejas con hijos y los hogares con jefe de menor nivel educativo. Cabe señalar que el aumento de la diferencia de ingresos tanto entre los mayores de 59 años y los niños y adolescentes, como entre hogares con jefe de distinto nivel educativo, venía verificándose desde mediados de la década de 1990, y que el proceso se profundizó con la crisis. Además, la concentración de los ingresos creció al interior de los grupos, reflejando una mayor homogeneidad de situaciones.

Para analizar las características socioeconómicas se utilizaron dos criterios: i) la fuente principal del ingreso del hogar y el nivel educativo del jefe de hogar, y

APÉNDICE

\section{Los datos utilizados}

Los datos que se utilizaron provienen de la Encuesta Continua de Hogares (ECH) que lleva a cabo el Instituto Nacional de Estadística (INE) de Uruguay, y corresponden especialmente a los años 1998 a 2002.

La ECH es una encuesta urbana ponderada, representativa de los hogares residentes en localidades con más de 5.000 habitantes. Recopila información sobre características personales, laborales y de ingresos. Da a conocer el ingreso de todos los integrantes del hogar, distinguiendo entre los que provienen del trabajo, del capital y de transferencias. En todos los casos se informa el ingreso neto percibido, esto es, el monto registrado luego de pagar los impuestos. ii) el tipo de actividad de los perceptores de ingreso. Con ambos se recogieron importantes cambios en la participación de los grupos socioeconómicos en la población: se elevó el porcentaje de personas en hogares de trabajadores con al menos un desocupado y aumentó el de personas en hogares que combinaban múltiples fuentes de ingresos. Estos cambios reflejan los problemas de empleo, que tuvieron un impacto concentrador de signo positivo sobre la desigualdad, aunque el efecto neto de los traspasos fue relativamente bajo.

A la vez, si bien todos los grupos sufrieron caídas de ingreso, las distancias entre los ingresos de los distintos grupos crecieron, con el consiguiente efecto concentrador. El análisis de los ingresos permite señalar cuatro fenómenos de importancia.

En primer lugar, en la clasificación por tipo de perceptor, la caída del ingreso per cápita medio fue más intensa para los hogares de trabajadores que para los de jubilados y pensionados. En segundo lugar, dentro del grupo de los dependientes de remuneraciones del trabajo, crecieron las brechas entre hogares con jefes de diferente nivel educativo. Recuérdese que en el mercado de trabajo tuvieron lugar algunos cambios que pueden contribuir a explicar ese distanciamiento: por un lado, el desempleo afectó en mayor medida a los de menor nivel educativo; por otro lado, continuó el aumento de las diferencias salariales entre trabajadores de distinta calificación. En tercer lugar, las personas que vivían en hogares dependientes de ingresos del capital soportaron una pérdida importante en términos relativos, lo cual puede asociarse a la crisis financiera. Por último, tendió a ampliarse la diferencia entre los ingresos de las personas en hogares con al menos un desocupado y aquellos de otros grupos.

En el 2001, el INE introdujo una modificación en el formulario y, en particular, comenzó a recoger de manera más detallada datos sobre los distintos rubros de ingreso. Esta modificación mejoró la recopilación de datos sobre los siguientes beneficios sociales: hogar constituido, asignación familiar y contribución para la atención de la salud. Como resultado, la proporción de perceptores de estos beneficios aumentó de $1,5 \%$ en el 2000 a $21,9 \%$ en el 2001 y el monto real medio de beneficios por perceptor aumentó 50\%. La mejora también incidió en las medidas de desigualdad, tendiendo a desconcentrar los ingresos. Por ello, para comparar los cambios entre los años 1998 y 2002 se ha preferido trabajar con el ingreso sin los beneficios sociales. 
APÉNDICE

\section{Descomposiciones}

a) Descomposición entre los índices de entropía y dentro de ellos

Los índices de entropía de grado $0\left(E_{0}\right)$ y $1\left(E_{l}\right)$ de la distribución del ingreso per cápita entre personas corresponden respectivamente a:

$E_{0}=(1 / n) \Sigma_{i} \ln \left(\mu / y_{i}\right)$

$$
i=1, \ldots, n
$$

$E_{1}=\Sigma_{i}\left(x_{i}\right) \ln \left(n x_{i}\right)=(1 / n) \Sigma_{i}\left(y_{i} / \mu\right) \ln \left(y_{i} / \mu\right) \quad i=1, \ldots, n$

donde $y_{i}$ representa el ingreso per cápita correspondiente a la persona $i, \mu$ es el ingreso promedio de la población, y $x$ es la participación en el ingreso de la persona $i$.

Una propiedad que ha vuelto atractiva a esta familia de índices de desigualdad es que se pueden desagregar de forma aditiva en dos componentes que dan cuenta de la contribución a la desigualdad total que hace la desigualdad dentro de grupos y entre grupos de la población. Así:

$E_{0}=\left\{\Sigma_{g}\left[\left(n_{g} / n\right)\right] E_{0 g}\right\}+\left\{(1 / n) \Sigma_{g} n_{g} \ln \left(\mu / \mu_{g}\right)\right\}$

$E_{1}=\left\{\Sigma_{g}\left[\left(n_{g} / n\right)\left(\mu_{g} / \mu\right)\right] E_{l g}\right\}+\left\{(1 / n) \Sigma_{g} n_{g}\left(\mu_{g} / \mu\right) \ln (\mu / \mu)\right\}$

Obsérvese que el primer término es la suma ponderada de los índices de entropía de cada grupo, por lo que su valor es una medida de la contribución del grado de concentración dentro de los grupos a la desigualdad total. La ponderación viene dada en el caso de $E_{0}$ por la participación de cada grupo en la población y en el caso de $E_{1}$, por la participación en el ingreso total. A su vez, el segundo término es el valor del índice calculado para los ingresos medios de cada grupo. Así, este componente puede ser interpretado como una medida de la desigualdad en que se ha supuesto que todas las personas de un grupo tienen el mismo ingreso per cápita, concentrando la atención en las diferencias entre los grupos.

Esta descomposición permite medir el poder explicativo de una clasificación, ya que cuanto mayor sea la contribución porcentual del componente más potente será la clasificación realizada para explicar la desigualdad total.

\section{b) Descomposición en el tiempo de los cambios experi-} mentados por la desigualdad total

La desigualdad agregada puede variar por tres motivos. En primer lugar, por modificaciones en la distribución dentro de los grupos: cuando la desigualdad en un grupo crece, hay un efecto concentrador sobre la población total. En segundo lugar, los cambios en la participación de cada grupo también influyen sobre la desigualdad total: un traspaso de personas desde el grupo con menor dispersión al más desigual tiene un efecto concentrador. Además, los cambios en la participación de los grupos afectan la relación entre el ingreso promedio de cada grupo y el ingreso promedio total: así, tras- pasos de personas de los estratos medios hacia los altos o bajos tiene un impacto concentrador. En tercer lugar, las variaciones de los ingresos promedio también afectan la distribución, ya que los aumentos de las diferencias de ingresos entre grupos llevan a una mayor desigualdad.

En el análisis de los cambios por origen de los ingresos se utilizó la descomposición de Mookherjee y Shorrocks (1982), la que permite desagregar la variación del índice de entropía 0 entre el año $t$ y $t+i$ en cuatro componentes, para conocer el efecto que tienen los cambios en el origen de los ingresos sobre la desigualdad agregada. Así, los autores proponen utilizar la siguiente aproximación:

$$
\begin{gathered}
\Delta E_{0} \approx \Sigma_{g} \overline{v_{g}} \Delta E_{0, g}+\Sigma_{g} \overline{E_{0, g}} \Delta v_{g}+ \\
\Sigma_{g}\left(\overline{\lambda_{g}}-\overline{\ln \lambda_{g}}\right) \Delta v_{g}+\Sigma_{g}\left(\overline{\theta_{g}}-\overline{v_{g}}\right) \Delta \ln \mu_{g}
\end{gathered}
$$

donde $v_{g}$ es la participación en la población, $\lambda g$ es la relación entre el ingreso per cápita medio del grupo y el ingreso per cápita medio total y $\theta g$ su participación en el ingreso total.

El primer sumando (término A) responde a los cambios puros de la desigualdad dentro de cada grupo; los siguientes dos sumandos (términos B y C) a cambios en la estructura de los grupos y el último (término D) a las variaciones de sus ingresos medios. Para cada término, los signos positivos indican un impacto concentrador, ya que contribuyen a aumentar el índice de entropía general.

Un análisis más detallado de cada uno de los términos pone de manifiesto la potencia de esta propuesta metodológica.

El término A es la suma ponderada de la variación de los índices de entropía de cada grupo. Los ponderadores son positivos y su suma es idéntica a la unidad. Por lo tanto, cuando crece la desigualdad al interior de los grupos, el término A tiene un impacto concentrador y su magnitud dependerá de la participación de los grupos en la población. Si el aumento de la desigualdad total se debe fundamentalmente a este término, entonces el origen del ingreso de las personas habrá perdido poder explicativo en sentido estricto, ya que otras variables que no han sido consideradas en la clasificación habrán adquirido importancia durante el período.

El término B es otro de los componentes de la variación de la desigualdad dentro de los grupos, pero responde a los cambios en la participación de las personas y se calcula como su suma ponderada por los índices de entropía de cada grupo. Obsérvese que la suma de $\Delta v_{g}$ es nula, por lo que el signo del término $\mathrm{B}$ depende altamente del valor de los ponderadores. Por ejemplo, si la población estuviera clasificada en dos grupos, el término tendría signo positivo cuando existiera un traspaso de personas desde el grupo con menor desigualdad interna al de mayor concentración. En 
términos generales, entonces, los cambios en la composición de los grupos debidos a un aumento de la participación de aquellos con menor equidad interna tendrán un impacto concentrador sobre la desigualdad total.

El término $C$ recoge también cambios en la estructura de los grupos, pero en este caso, el ponderador de la variación de las participaciones es una función parabólica del ingreso medio relativo, cuyo valor mínimo corresponde a $\left(\lambda_{g}, \lambda_{g}-\ln \lambda_{g}\right)=(1,1)$. Por lo tanto, los traspasos de personas de estratos de ingreso medios a estratos altos y/o bajos se sintetizarán en un signo positivo del término C. Intuitivamente, es fácil aceptar que la disminución del número de personas con ingresos similares al promedio tienda a aumentar el poder explicativo de las diferencias entre grupos y tenga un impacto concentrador sobre la desigualdad global.

Por último, el término D es la suma ponderada de las variaciones del ingreso medio de los grupos (en logaritmos). Obsérvese que el ponderador $\theta_{g}-v_{g}$ es positivo cuando el ingreso medio del grupo es superior al promedio. En este caso, si el ingreso del grupo crece, su efecto sobre D es positivo. En cambio, si $\theta_{g}-v_{g}$ es negativo, el crecimiento del ingreso del grupo tiene un impacto negativo. Como la suma de los ponderadores es cero, el resultado final del término D dependerá de la medida en que se compensen los efectos de los distintos grupos. Por lo tanto, un signo positivo del término indica que los cambios en los ingresos medios contribuyeron a acrecentar el grado de desigualdad entre personas como resultado de un aumento de las diferencias medias entre los grupos.

\section{Bibliografía}

Amarante, V. y R. Arim (2003): Mercado laboral en Uruguay, 1986-2002, documento presentado en las Jornadas sobre Empleo, octubre 2003, organizadas por el Centro Interamericano de Investigación y Documentación sobre Formación Profesional (CINTERFOR) de la Organización Internacional del Trabajo (огт) y la Universidad de la República, Montevideo, octubre.

Arim R. y G. Zoppolo (2000): Remuneraciones relativas y desigualdad en el mercado de trabajo. Uruguay: 1986-99, monografía presentada en la Facultad de Ciencias Económicas y Administración de la Universidad de la República, Montevideo.

Arim R. y M. Furtado (2000): Pobreza, crecimiento y desigualdad. Uruguay 1991-97, serie documentos de trabajo D.T 5/00, Montevideo, Instituto de Economía, Facultad de Ciencias Económicas y Administración, Universidad de la República.

Bucheli, M. y C. Casacuberta (2003): La estructura del desempleo en Uruguay,1991-2002, documento de trabajo presentado en las Jornadas sobre Empleo, organizadas por el Centro Interamericano de Investigación y Documentación sobre Formación Profesional (CINTERFOR) de la Organización Internacional del Trabajo (огт) y la Universidad de la República, Montevideo, octubre.

Bucheli, M. y M. Furtado (2000a): La contribución de las distintas fuentes de ingreso a la evolución de la desigualdad en el Uruguay urbano, 1986-1997, LC/MVD/R.183, Montevideo, Oficina de la CEPAL en Montevideo.

(2000b): La evolución de la participación de las fuentes de ingreso en Uruguay 1986-1997, documento presentado en el Seminario sobre distribución del ingreso y pobreza, orga- nizado por el Departamento de Economía de la Facultad de Ciencias Sociales de la Universidad de la República, Montevideo.

Bucheli M. y M. Rossi (1994): Distribución del ingreso en el Uruguay, documento de trabajo, $\mathrm{N}^{\circ} 10 / 94$, Montevideo, Departamento de Economía, Facultad de Ciencias Sociales, Universidad de la República.

Casacuberta, C. y M. Vaillant (2002): Trade and wages in Uruguay in the 1990's, documento de trabajo, $\mathrm{N}^{\circ} 13 / 02$, Montevideo, Departamento de Economía, Facultad de Ciencias Sociales, Universidad de la República.

Kaztman, R., F. Filgueira y M. Furtado (2000): Nuevos desafíos para la equidad en el Uruguay, Revista de la CEPAL, $\mathrm{N}^{\mathrm{0}} 72$, LC/G. 2120-P, Santiago de Chile, diciembre.

Machado A. e I. Reggio (1999): Incidencia de la reforma en el mecanismo de ajuste de las pasividades de 1990 sobre la distribución del ingreso de los hogares. Uruguay: 1986-1997, monografía presentada en la Facultad de Ciencias Económicas y Administración, Universidad de la República, Montevideo.

Miles D. y M. Rossi (2001): Wage inequality in developing countries: market forces or government intervention, inédito.

Mookherjee, D. y A. Shorrocks (1982): A decomposition analysis of the trend in UK income inequality, The Economic Journal, vol. 92, Oxford, Reino Unido, Blackwell Publishing.

PNUD (Programa de las Naciones Unidas para el Desarrollo) (2001): Informe de desarrollo humano en Uruguay, Montevideo.

Vigorito, A. (1999): Una distribución del ingreso estable. El caso de Uruguay 1986-1997, Revista de Economía, vol. 6, No 2 , Montevideo, Banco Central del Uruguay. 